Participation Seven Years after Severe Childhood Traumatic Brain Injury: Results of the TGE Prospective Longitudinal Study

Hugo Câmara-Costa ${ }^{* 1,2}$, Leila Francillette ${ }^{2}$, Marion Opatowski ${ }^{3}$, Hanna Toure ${ }^{4}$, Dominique Brugel $^{4,5}$, Anne Laurent-Vannier ${ }^{4,5}$, Philippe Meyer ${ }^{6,7}$, Georges Dellatolas ${ }^{1}$, Laurence Watier $^{3}$, Mathilde Chevignard ${ }^{2,4,5,8}$

1 Université Paris-Saclay, Université Paris-SUD, UVSQ, CESP, INSERM, Paris, France

2 Sorbonne Université, Laboratoire d'Imagerie Biomédicale (LIB), Paris, France

3 Biostatistics, Biomathematics, Pharmacoepidemiology and Infectious Diseases (B2PHI), INSERM, UVSQ, Institut Pasteur, Université Paris-Saclay, Paris, France

4 Rehabilitation Department for Children with Acquired Brain Injury, Hôpitaux de Saint Maurice, Saint Maurice, France

5 Outreach team for Children and Adolescents with Acquired Brain Injury, Hôpitaux de Saint Maurice, Saint Maurice, France

6 Paediatric Anesthesiology Department, Hôpital Necker Enfants Malades, Paris, France

7 Faculté de Médecine René Descartes, Université Paris 5, Paris, France

8 Groupe de Recherche Clinique Handicap Cognitif et Réadaptation (HanCRe) Sorbonne Université, Paris, France

* Corresponding author: CESP INSERM U1018, 97, boulevard de Port Royal, 75014 Paris, France. Email: hugocamaracosta@gmail.com; Tel.: +33(0)646534466; Fax. $+33(0) 158412843$.

Running head: Participation 7 Years Post Severe TBI 


\title{
Participation Seven Years after Severe Childhood Traumatic Brain Injury:
}

\begin{abstract}
Purpose: Participation in home, school and community activities is considered as the ultimate aim of rehabilitation. The aims of this study were to examine participation seven years post-severe childhood traumatic brain injury and factors associated with participation.
\end{abstract}

Materials and methods: Participants were enrolled in the Traumatisme Grave de l'Enfant (Severe Childhood Injury) cohort study following severe accidental childhood traumatic brain injury. Participation seven years post-injury, was examined using parent- and self-report forms of the Child and Adolescent Scale of Participation among 37 patients [62\% males, mean age 15.4 years $(S D=4.4)$, mean length of coma 6.68 days $(\mathrm{SD}=4.96)]$ and 33 matched controls.

Results: Parent reports indicated significantly lower participation among patients compared to controls, but the self-reports did not. In the traumatic brain injury group, parent-reported participation was variable, with $22 \%$ of the patients clearly showing greater restrictions than controls. Participation restrictions were significantly associated with injury severity, poor functional outcome one-year post-injury, executive and behavioral difficulties and higher fatigue levels seven years post-injury, but not with pre-injury nor family factors.

Conclusions: Several years after severe childhood traumatic brain injury, participation appears to depend more on injury-related factors than on environmental factors. In selfreports assessments of participation, it could be difficult for children and adolescents to distinguish capacity from performance.

Keywords: participation, severe traumatic brain injury, outcome, child, prospective cohort study 


\section{Introduction}

Traumatic brain injury (TBI) is a global public health concern. The worldwide incidence of pediatric TBI ranges from 47 to 280 per 100000 children in most reports. Severe pediatric TBI, defined by a Glasgow Coma Scale (GCS) score $\leq 8$, accounts for $3 \%$ to $7 \%$ of all pediatric TBIs, and is much less frequent than mild TBI (GCS >13), which accounts for more than $80 \%$ of pediatric TBIs [1].

Several investigations report chronic social impairment after severe pediatric TBI, since the brain regions involved in social cognition are often the most impacted after head trauma $[2,3]$. According to a systematic review of the literature, only children and adolescent survivors of moderate-to-severe TBI (but not mild TBI) present an elevated risk of social dysfunction [4]. Psychosocial outcome was found to be better after mild compared to moderate-severe TBI [5], or after moderate compared to severe TBI $[3,6]$.

Children with severe TBI demonstrate selective, long-term deficits in their social problemsolving skills, associated with poor social and academic outcomes [7], social withdrawal [8], reduced social cognition related to diffuse neuropathology and parietal lesions [9], poor adaptive functioning linked to deficits in fluid reasoning and processing speed deficits [10], social impairment associated with cognitive control deficits [11], higher risk of peer victimization [12], or reduced leisure time in physical activity related to balance deficits [13]. Social outcome after severe TBI depends on injury-related characteristics, as well as environmental factors, and there is some debate about their respective role. Some studies suggest that social impairment after pediatric TBI is mainly related to environmental factors, such as family functioning, and less to injury factors, such as severity [14-16]. In contrast, other reports indicate that psychosocial outcome following severe pediatric TBI may be less responsive to environmental factors (family resources and stressors) than mild-to-moderate pediatric TBI $[17,18]$. 
Participation has been considered as the ultimate aim of rehabilitation $[19,20]$, and its measurement after pediatric TBI has been recommended [21]. Nevertheless, there is some debate about how participation should be defined and conceptualized for individuals with disabilities, and particularly for children with disabilities. The International Classification of Functioning, Disability and Health (ICF) [22] defines participation as "involvement in a life situation", and participation restrictions as "problems an individual may experience in involvement in life situations". These definitions do not clearly differentiate activity from participation. For Whiteneck and Dijkers [23], activities are the functional performances of an individual in isolation (individual functioning), while participation is a relational concept (societal functioning). Self-care, for instance (e.g. toileting, dressing, eating), includes activities that would not be regarded as social [24]; conversely, interpersonal interactions, maintaining relationships and generating social roles add up to participation. It should be noted, however, that self-care itself can imply a relational aspect (with caregivers) when an individual is unable to perform certain self-care activities as a consequence of age, in the case of very young children, as noted by Coster and Khetani [25], or severe impairment. Thus, inability to perform self-care activities might be conceptualized as negative participation. For these authors [25], the distinction between activity and participation calls on complex concepts rather than on the mere presence or absence of a relational aspect: activities are specific tasks and actions that are simpler than "life situations", which are "sets of organized sequences of activities directed toward a personally or socially meaningful goal" (pp. 643). There is more agreement for a distinction between capacity: what a child can do in an ideal environment, and performance: what a child actually does in his/her environment [24]. Differences between capacity and performance can arise from environmental and personal factors. Environmental factors include availability of products and technology, barriers and facilitators in natural and human-made environment, support and relationships, attitudes, and 
services and policies; personal factors include idiosyncratic preferences and choices [20,26]. Further to this, objective indicators of participation should be distinguished from subjective aspects of participation, which relate to the meaning and the importance attached by an individual to participation in particular life situations. These subjective aspects include the sense of belonging and satisfaction with life situations, and have been considered to be aspects of Quality of Life [25]. Finally, according to McConachie [24], measuring participation among disabled children needs to consider their dependency on the family and their changing abilities and autonomy as they grow older.

Overall, the above considerations suggest that participation has multiples determinants: (i) individual factors determining capacity, such as physical and cognitive impairments; (ii) individual factors affecting performance in the absence of incapacity, such as psychological and behavioral factors deriving from attitudes, preferences and choices; (iii) family-related factors, especially among young children; and (iv) other environmental and cultural factors. Among the proposed measures of participation, Bedell and Coster [19] distinguish (i) measures that explicitly assess participation, such as the Children's Assessment of Participation and Enjoyment (CAPE), the participation subscale of School Function Assessment (SFA), the Assessment of Life Habits for Children (LIFE-H), and the Child and Adolescent Scale of Participation (CASP); (ii) subsections in other measures that implicitly assess participation, such as the Vineland Adaptive Behavior Scales, the Child Behavior Checklist (CBCL), and the Child Health Questionnaire (CHQ); and (iii) tailored or personalized measures assessing person-centered or intervention-specific goals and/or measures using observation and interviews.

The present study used the CASP, which includes four subsections: participation at home, in the neighborhood and community, at school, and in home and community living activities [27,28]. An important question is whether or not participation, as measured with the CASP, 
should be considered as a unidimensional trait that can be assessed by a single score. Overall, studies using the CASP were in favor of a relatively strong unidimensional structure. However, the findings pertaining to the exploration of possibly distinct dimensions varied across studies, and seemed to depend strongly on differences in the samples explored: exclusively children and young people with disabilities, or participants with and without disabilities; differences in the disabilities' severity level; proportions of participants with ceiling ratings for participation; and proportions of participants with communication difficulties, mobility restrictions, cognitive deficits, among others [27-35].

Assessments of participation could be more appropriate when children are questioned directly [24]. The CASP proposes a self-report version in addition to the parental report. The factorial structure of the two reports was similar and the young people reported significantly higher levels of activity/participation than did their parents [36].

A review of participation after pediatric $\mathrm{ABI}$ reported evidence of participation restrictions in home, in school and in the community 18 months after the occurrence of the injury [37]. Reported factors associated with participation restrictions include injury severity markers, motor, behavioral and cognitive difficulties, as well as family functioning, accessibility, and household income [34]. For parents of children with ABI, participation depends on the child's motivation, supportive relationships from family and friends, and supportive community attitudes [38]. Parents explicitly link participation to environmental factors [39]. Previous studies using the CASP have reported reduced participation among children and young people after severe TBI compared to controls or to children with mild TBI [35,40-45]. However, in these studies the number of children with severe TBI was generally small and, in some studies, moderate and severe TBIs were pooled. In addition, participation rates were relatively high in some reports, even after severe TBI (e.g. [42]), suggesting that only a proportion of 
participants, such as those with more severe impairments, may suffer participation restrictions.

The aims of the present study were (1) to investigate participation using the CASP self- and parent-reports among children and young adult survivors of severe childhood TBI, included in the prospective longitudinal cohort study Traumatisme Grave de l'Enfant (TGE; Severe Childhood Injury), seven years after injury, in comparison with ratings obtained in a closely matched control group; and (2) to determine factors influencing participation outcomes (among socio-demographic, TBI severity-related, and concurrent TBI outcomes factors). Participation was treated as a unidimensional trait. Participants with and without participation restrictions, defined on the basis of the ratings of the control group, were compared on sociodemographic and injury-related factors, pre-injury and family functioning, as well as on functional, cognitive, behavioral, educational and quality of life outcomes.

\section{Materials and methods}

\section{Participants}

\section{Patients with TBI}

The participants included were children aged 0-15 years consecutively admitted within 6 hours following severe accidental TBI to the pediatric neurosurgical intensive care unit (ICU) at Necker Enfants Malades Hospital between January 2005 and December 2008. Eighty-one children were included at the acute stage of TBI, defined as a Glasgow Coma Scale (GCS) score $\leq 8$ [46] at admission and/or an Injury Severity Score (ISS) $>16$ [47]. The causes of the accidental TBIs were motor vehicle accidents and falls. The exclusion criteria were: no vital signs at admission, non-accidental head injury, and previous history of diagnosed neurological, psychiatric or learning disorders. Of the 81 children initially enrolled, 16 died in acute care, leaving 65 children available for follow-up. All children received treatment according to international guidelines for the management of severe TBI [48] in the 
neurosurgical ICU of a regional pediatric trauma center, and most children (83\%) received inpatient multidisciplinary rehabilitation after acute care [49]. Follow-up comprised comprehensive medical and neuropsychological assessments at 3,12, 24 months and 7 years post-injury.

At 7 years post-TBI, 26 of the 65 patients were lost to follow-up or did not wish to participate, leaving 39 patients (aged 7-22 years) for assessment. Because two patients had no available participation ratings, the final sample comprised 37 patients. The participants followed at 7 years post-injury did not differ significantly from those lost to follow-up for the GCS score, the ISS score, length of coma, parental education, language used at home, or age at injury, nor for 3-, 12- or 24-months post-injury intellectual (Wechsler's Full Scale Intelligence Quotient) and executive functioning (Behavior Rating Inventory of Executive Function).

\section{Controls}

A population-based group of healthy controls was recruited from local schools or via general medical practices at the 7-year follow-up point. Controls were matched to participants in the TBI group for age ( \pm 3 months), gender and parental education ( \pm 2 years' education). The exclusion criteria were those used for the TBI sample, plus presence of a history of TBI. Among the 38 controls recruited for the study, 33 had available data on the CASP and were considered for the analyses reported here.

\section{Measures}

\section{Socio-demographic and pre-injury education}

Information on children's age, sex and family characteristics was collected in the initial phase, and reconfirmed at the time of the 7-year follow-up. We defined parental educational level as low (neither of the parents had a secondary school diploma [“baccalauréat"]) or as medium-high (at least one of the parents had a secondary school diploma). Family situation 
was dichotomized in two categories: child living with both biological parents or not (nonmarital or single parent status).

We generated a binary variable for the type of pre-injury education: Regular education referred to participants enrolled in mainstream school, with neither classroom support nor repeat school years; or Aided and/or Delayed, characterizing children enrolled in mainstream school, but having had classroom support and/or having repeated one or more years.

\section{Initial injury severity}

TBI severity was assessed using three classic quantitative scales designed to assess trauma severity, namely the Glasgow Coma Scale (GCS) [46], the Pediatric Trauma Score (PTS) [50] and the Injury Severity Score (ISS) [47,51,52].

\section{Outcome measures collected during follow-up}

\section{One-year post-injury}

Overall level of disability was measured with the Glasgow Outcome Scale modified for children (GOS Peds) [53], which aims at assessing global qualitative outcome among children and adolescents subsequent to TBI. The overall outcome is divided into 5 categories: (I) good outcome; (II) moderate disability, including hemiparesis and/or cognitive impairments and/or referral for outpatient rehabilitation therapy; (III) severe disability, including severe motor deficit and/or cognitive assessment in the deficient range and/or referral for inpatient rehabilitation; (IV) minimally responsive or vegetative state, and (V) death.

Functional outcome was measured using the Pediatric Injury Functional Outcome Scale (PIFOS) [54], a brief injury-specific multidimensional rating scale completed by parents and validated for children aged 3 to 15 years. This assessment was performed by a trained health care provider in a structured interview assessing a broad range of cognitive, physical, and psychological health areas commonly impacted by pediatric injury in children. When no impairment is reported by parents, in comparison with same-age peers in a specific domain, 
the item is rated 0 . Scores from 2 to 4 correspond to mild, moderate and severe levels of impairment respectively. Raw summary scores are derived, with higher scores indicating higher levels of functional impairment.

\section{7-years post-injury}

The Overall level of disability was measured using the GOS-Extended (Pediatric [GOS-E Peds] and Adult version [GOS-E]), a validated structured interview assessing TBI outcome among children, adolescents [53] and adults [55]. The outcome scores yield 8 categories: 1=Upper Good Recovery, 2=Lower Good Recovery, 3=Upper Moderate Disability, 4=Lower Moderate Disability, 5=Upper Severe Disability, 6=Lower Severe Disability, 7=Vegetative State and $8=$ Death.

Motor deficits: Neurological and functional assessment enabled the collection of information relating to the presence or absence of (1) hemiplegia or hemiparesis; (2) signs of cerebellar dysfunction (ataxia and/or coordination disorders). Motor deficits were categorized as the absence or presence of at least one of the above-mentioned deficits 7 years post-injury.

On-going education: We categorized on-going education according to two levels. Mainstream education referred to participants enrolled in general education classrooms, independently from the presence of classroom support and/or repeat year(s), and specialized education pertained to participants enrolled in establishments providing special education facilities, such as specialized or medico-educational schools.

Intellectual ability was measured using the age-appropriate French versions of the Wechsler Intelligence scales, namely: Wechsler Intelligence Scale for Children-IV (WISC-IV) [56] and Wechsler Adult Intelligence Scale-IV (WAIS-IV) [57]. The Full Scale Intellectual Quotient (FSIQ) was used. 
Executive Functioning was measured using the French adaptation and standardization of the parent-report of the Behavior Rating Inventory of Executive Functions (BRIEF) [58] for patients aged up to 17 years 11 months. The self-report of the Adult-BRIEF questionnaire [59] was also used for those aged 18 years and above, but results are not presented here due to the small number of participants. Several aspects of behavior related to executive functioning in everyday life are assessed through 86 items, distributed across eight clinically and theoretically-driven subscales measuring different aspects of executive functioning, and yielding two composite indices and a total score, used in the present study: the Behavioral Regulation Index (BRI), the Metacognition Index (MI), and the Global Executive Composite index (GEC) expressed as $T$-scores (mean $=50, \mathrm{SD}=10)$. Higher scores indicate poorer executive functioning.

Behavior was measured using the Child Behavior Checklist (CBCL) [60], a parental questionnaire aiming to assess children's behavioral problems from ages 4 to 18 years. This questionnaire enables the computation of 3 summary age-standardized scores ( $T$ scores with mean=50, $\mathrm{SD}=10$ ), specifically Internalizing problems, Externalizing problems and a Total problems score. Higher scores reflect more marked behavior problems.

Health-related Quality of life (HRQoL) was assessed using the age-appropriate Frenchvalidated versions of self- and parent-report forms of the Pediatric Quality of Life Inventory (PedsQL) for children (ages 2-18 years) [31] and young adults (ages 18-25 years) [61]. The Total Score, expressed on a 0-100 scale, was used in this study, with higher scores indicating better HRQoL.

Fatigue was assessed using the self- and parent-report forms of the Multidimensional Fatigue Scale (MFS) for children (ages 2-18 years) [62] and young adults (ages 18-25 years) [63]. The MFS is a symptom-specific module of the PedsQL aiming to assess fatigue across paediatric 
populations [64-66]. We used the total score and, similarly to the PedsQL, higher scores indicate lower reports of fatigue.

Family functioning was assessed using the 12-item short form of the French version of the Family Assessment device (FAD) [67]. This questionnaire assesses family functioning over six domains (Problem Solving, Communication, Roles, Affective Responsiveness, Affective Involvement, and Behavioral Control). We used the total score (range 1 to 4), with higher scores indicating worse family functioning.

\section{Primary outcomes 7-years post-injury}

Child and Adolescent Scale of Participation (CASP):We used the French version of parent and self-report forms of this questionnaire aimed at assessing the degree of participation of children in home, school and community activities compared to children with the same age [27,28]. This questionnaire comprises 4 subscales: Home Participation (items 1 to 6 ), Community Participation (items 7 to 10), School Participation (items 11 to 15) and Home and Community Living Activities (items 16 to 20). Each item is rated on a four-point scale: (1) Unable; (2) Very Restricted; (3) Somewhat Restricted; and (4) Age Expected (Full participation). A "Not Applicable" rating is also available for activities in which the child is not expected to participate. The subscales and the Total scores are obtained by summing the ratings of the "Applicable" items. These scores are then converted into a 100-point scale by dividing the sum of the "Applicable" items by the maximum score possible (based on the total of "Applicable" items that were rated) and multiplying it by 100. Lower levels of participation correspond to lower scores. In the present study, internal consistency coefficients were good, with Cronbach alpha for parent-reports of .74 and .84 for controls and participants with TBI respectively, and .81 and .87 for self-reports.

The parent-report was used for the whole sample and the self-report was given to participants aged 11 years or above. 


\section{Procedure}

This study was approved by the (Comite de Protection de Personnes d'Île-de-France VI [CPP IDF VI]) ethics committee and parents gave their informed written consent to participate in the study. Patients (parents for those under 18 years of age) who had been included in the TGE study were contacted by the treating physician assessing them in the initial phase of the study and asked to participate in the 7-year follow-up. The assessments took place in the Rehabilitation Department in the Saint Maurice Hospitals, on two separate occasions. A physician specialized in Physical Medicine and Rehabilitation performed the medical assessment, which included information about ongoing medications, care and rehabilitation therapies, type of ongoing education and current occupation, GOS-E or GOS-E Peds rating scales, and a neurological examination assessing the presence of motor, visual or hearing impairments. In a different day, a trained child psychologist performed the neuropsychological assessment, and administered the self-version questionnaires in a fixed order, while parents answered the proxy-versions of the questionnaires. For the control group, the data collected during this period was the same as for the TBI group, although controls did not undergo the medical examination and only performed a reduced number of the neuropsychological assessments administered to patients.

\section{Statistical analyses}

Data analysis was performed using the SAS@ software version 9 [68]. We used chisquare tests and non-parametric univariate procedures (Wilcoxon signed-rank test) to compare TBI participants and controls according to parent and self-reported participation and 7-years post-injury outcomes (on-going education, FSIQ, BRIEF, CBCL, PedsQL, MFS and FAD). These analyses were performed using the total number of observations available for each instrument. Missing data was related to participants and/or parents not returning one or several of the proposed questionnaires. 
We used the lowest score observed for the control group in the parent-report version of the CASP (82.5) as a cut-off to define two subgroups of patients with TBI: a subgroup with participation scores in the control group range (score $\geq 82.5$ ) and a subgroup with participation restrictions (score < 82.5). These two subgroups were compared on age at injury, preinjury education, initial injury severity, and 1-year and 7-years post-injury outcomes, using the Wilcoxon signed-rank and chi-square tests. In addition, we used linear regressions (repeated measures Generalized Lineal Model) to investigate differences between participation subscales according to the type of domain assessed, as well as the group (TBI vs. controls) by informant (parent $v s$. self-reports) interaction. Statistical significance testing was 2-tailed with a $p<.05$ as the significance level

\section{Results}

\section{Participants with pediatric severe TBI (Table 1)}

The analysis sample was composed of 37 patients with severe pediatric TBI (23 males, 63\%), mean age at injury was 7.62 years (range $.25-14.67$; median 7.67 years). Injury severity was characterized by: mean lowest GCS score 5.92 (range 3-8; median 6), mean PTS score 3.97 (range -1 to 9; median 4), mean ISS score 27.76 (range 4-50; median 29) and mean length of coma 6.68 days (range 1-22 days; median 5 days). Prior to their injury, 5 participants had received school assistance and/or had repeated one year.

At 1-year post-injury, the GOS-Peds scores of 5 participants (14\%) were in the good recovery range, $20(54 \%)$ were in the moderate disability level and $12(32 \%)$ in the severe disability level. The functional outcome (PIFOS) mean score was 26.74 (range 4-73; median 25). At 7 years post-injury, the GOS-E and GOS-E Peds scores were as follows: 20 participants (54\%) were in the good recovery range (Upper [ $n=4]$ and Lower levels $[n=16]$ ); overall disability was moderate for 9 (24\%) participants (Upper [ $n=5]$ and Lower levels [ $n=4]$ ); and 8 
participants (22\%) were in the severe disability range (Upper [ $n=5]$ and Lower levels $[n=3]$ ). The presence of motor deficits 7 years post-injury was reported for 7 (19\%) participants.

[INSERT TABLE 1 HERE]

\section{Group comparisons between participants with severe TBI and controls}

Socio-demographic characteristics and 7-year follow-up outcomes (Table 2)

The participants from the TBI group were similar to controls for socio-demographic characteristics, namely age, sex, parental education level and family situation.

At 7-years post-injury, fewer participants from the TBI group were enrolled in mainstream education than among controls. The TBI group presented significantly lower FSIQ scores, higher BRIEF scores on the BRI, MI and GEC (greater executive function problems reported by parents), higher mean CBCL scores (more marked behavioral problems reported by parents) on the Externalizing problems scale and the Total score, and lower mean scores pertaining to HRQoL and fatigue (lower quality of life and higher fatigue levels both for parent and self-reports). There was no significant difference between groups regarding family functioning.

\section{[INSERT TABLE 2 HERE]}

\section{Participation (Table 3)}

In comparison to the control group, the mean participation scores obtained from parentreports in the TBI group were significantly lower for all the CASP subscales and the Total score. The lowest mean parental score for the TBI sample was observed in the Home and community living activities subscale, and the results of the repeated measures analyses showed that mean scores differed according to the participation domain evaluated by parent-reports $(\mathrm{F}(3,29)=10.57, p=.004)$.

\section{[INSERT TABLE 3 HERE]}


As presented in figure 1, 18 (49\%) participants from the TBI sample presented participation scores in the 90 to 100 range, in comparison to $27(87 \%)$ participants from the control group. The lowest parent-reported participation Total score observed in the control group was 82.5 , while 8 participants $(22 \%)$ in the TBI group had scores inferior to 82.5 .

\section{[INSERT FIGURE 1 HERE]}

For self-reports, the results of the repeated measures analyses showed that the subscale mean scores differed according to the participation domain evaluated $(\mathrm{F}(3,19)=4.11$, $p=.02$ ), but there were no significant differences in the mean participation scores between the TBI sample and controls. Participation scores tended to be lower for proxycompared to self-reports in the TBI group, while the opposite pattern was observed in the control group (Informant by group interaction: $\mathrm{F}(1,49)=6.72, p=.0125)$.

\section{Factors associated with parent-reported participation in the TBI group}

According to parent-reports, the group of 8 participants with poor participation (i.e. CASP scores lower than 82.5), compared to the group of 29 participants with CASP scores in the range of the control group, presented:

i) higher injury severity indices: lower GCS, higher ISS scores and longer length of coma);

ii) poorer outcomes 1-year post-injury: increased overall disability (GOS-Peds) and poorer functional outcome (PIFOS);

iii) poorer outcomes 7-years post-injury: increased overall disability (GOS-E/GOS-E Peds), more frequent reports of difficulties in executive functioning (BRIEF Behavioral Regulation and Metacognition Indices), higher ratings for behavioral problems (CBCL Total score), lower parent and self-reported HRQoL, and greater fatigue levels. 
There were no significant associations between parent reported participation and age at injury, parental education level, family situation, PTS, presence of motor deficits, ongoing education, FSIQ, or family functioning.

\section{Discussion}

In the present prospective longitudinal study, we report self- and proxy-reports of participation in a large sample of individuals who sustained severe TBI in childhood, followed over a 7-year period. Overall, the findings indicate reduced proxy-rated (but not self-rated) levels of participation compared to those of a closely matched control group. Interestingly, in parent-reports, participation was generally lower than in self-reports from the TBI sample, whereas the opposite pattern was observed in the control group. In addition, a sub-group of participants with low participation levels was identified using the lowest participation score in the control group as a cut-off. This sub-group accounted for $22 \%$ of the whole sample, suggesting satisfactory participation levels for a majority of patients. Those with low participation levels were characterized by more severe outcomes in most domains explored, especially in the cognitive and behavioral domains, and also reported lower levels of quality of life and higher levels of fatigue.

On the other hand, self-reported participation did not show any significant difference between the TBI and control groups. This was related to a tendency in the TBI sample to self-rate their participation somewhat higher compared to their parents, as noted in a previous study on children with disabilities [36], but an opposite tendency was observed among controls, where self-rated participation tended to be lower than parental ratings for participation. While the first observation could be related to a degree of selfawareness deficit following TBI, the second was less expected. It is important to note that the mean percentage for reported participation of the control group in the present study (about 91\%) was lower than percentages reported after moderate-severe TBI in 
some earlier studies (e.g. >98\% in [42]). One possible explanation is that in the control group, the participants' answers are based more on preference/performance, with answers reflecting the attractiveness of the respondent for certain types of activities (e.g. sports, shopping, doing laundry, washing dishes), while parents base their answers more on capacity. In the severe TBI group, where capacity can be a real problem, this tendency to respond on preference/performance could be less pronounced. However, it might be difficult to discriminate in some cases whether participation restrictions are due to a problem of capacity or preference/performance, such as in the case of an apathy syndrome, defined as lack of motivation [69], which is reported to be relatively frequent after severe TBI [70,71].

Parent-reports, in contrast, showed lower mean participation rates compared to controls in children and young adults after severe TBI, 7 years post-injury. The differences between patients and controls were significant for all four domains assessed by the CASP. In the TBI group, participation scores were lower for "home and community living activities", than for "home participation" and "school participation" [72]. However, not all participants in the TBI group showed low levels in parent-rated participation, and the variability of the total CASP score was wide, as also reported by Aaro Jonsson [73]. The distributions of the total CASP scores showed that 8 participants (22\%) of the TBI group obtained scores lower than the "less participating" control.

In order to investigate the factors associated with participation 7 years after severe pediatric TBI, we compared the sub-group of 8 participants with clear parent-rated participation restrictions, and the group of 29 participants with a participation level in the range of controls. 
Age at injury (and age at assessment) were not associated with participation levels, in accordance with some previous studies using the CASP [28], but not with others [74]. Pre-injury school difficulties and current family functioning were not associated with participation. This finding contrasts with a number of previous reports on children after TBI of varying severity. However most of these studies included a majority of mild TBIs, where pre-injury factors and family functioning were associated with psychological functioning and participation [14-16,42]. This result is in favor of the hypothesis that severe pediatric TBI could be less responsive to environmental factors than mild-to-moderate pediatric TBI $[17,18]$.

Almost all previous reports using the CASP found lower participation among children after severe TBI than among controls [40,45,75], and strong associations of participation with functional outcome [76], despite some exceptions [77]. The present study adds information on this topic, showing that these associations remain significant within a group following severe TBI. Seven years after severe TBI, patients with participation restrictions, compared to those with participation rates within the range of the control group, had worse scores for most markers of initial injury severity (GCS, ISS, length of coma), poorer functional outcome levels (PIFOS) measured 1-year postinjury, and greater overall level of disability according to the Glasgow Outcome Scale at 1 year and 7 years post-injury. Regarding cognitive factors, executive functions, as assessed by the BRIEF, were lower in the participation-restrictions group, whereas FSIQ was not. In previous reports, processing speed [43] and communication skills [44] were found to be associated with participation after pediatric TBI. Fatigue, rarely assessed but reported to be "problematic" after pediatric TBI [73], and, as in other studies, quality of life (PedsQL) [29], and behavioral problems (CBCL) [35] showed significant links with parental reports of participation . Finally, special education was 
more frequently required in the "restrictions" group (37\% vs 17\%), but the difference did not reach significance.

Overall, the present findings from France show that participation as measured with the CASP parent-report is an interesting long-term outcome measure for patients who sustained severe childhood TBI. Despite the small number of participants with "participation restrictions", clearly significant associations were found with indices of injury severity, functional outcome, executive functions, behavioral difficulties, fatigue, and Quality of Life. For McConachie [24], an advantage of the CASP is its good basic coverage of the ICF domains, and a limitation is its emphasis on patients' ability to take part (on capacity), rather than on whether and how often they actually do (on performance). In favor of this assertion are the associations of the CASP total score with injury severity and functional outcome in parent-reports. It is likely that parents respond more on capacity than on performance, while, as noted previously, this is probably not the case in self-reports, at least for the control participants of the present study. It may be difficult for children and adolescents to make a clear distinction between capacity and performance (e.g. if I never washed dishes, I am definitely not able to do so). In addition, cultural factors and precise instructions on how to complete the CASP may influence the results, as suggested by strong differences of the mean scores and the proportion of participants with ceiling scores observed from one study/country to another.

The current results should be interpreted in the light of certain limitations. Despite the fairly large number of participants who sustained severe childhood TBI compared to previous studies, the overall sample size is nevertheless small and the comparisons of children with poor and normal levels of participation were performed on relatively small samples. The results do however highlight that a large proportion of participants report satisfactory 
participation levels following a severe childhood brain injury. In addition, our analyses were based on the total number of observations available for the primary outcome that differed across informants completing the questionnaires. For this reason, the sample size of the control group was smaller than that of the TBI group. Further to this, questionnaire-based reports of participation can be related to personal factors of the respondent, and reports of this type are subject to this bias, unlike objective measures of individuals' performance in everyday life. The advantage of using the CASP, however, is that it allows comparisons of results across studies and countries. In our sample, the levels of participation were relatively low overall in the control group, compared to participation levels of patients with various levels of TBI severity in other countries. This could be simply due to cultural factors, but also partially to the recruitment of the control group. Because controls had to be closely matched to patients, the inclusion criteria rendered this recruitment particularly selective, especially for the enrollment of participants from very low parental education backgrounds and very deprived socio-economic status. As a result, the control sample might not be representative of the French general population. However, this could also be considered as a strength of the present study, since many children with TBI come from low socioeconomic backgrounds. Finally, it would have been more informative to have measured participation using the same measure prospectively from the initial phase, in order to monitor change over time and factors associated with change. This was not possible as participation was not an outcome of interest in the first stage of the study. Further the CASP was not available at the time of the study commencement.

\section{Conclusions}

At the group level, participation is significantly impaired in the long term following pediatric severe TBI, mostly in parent-reports. However, participation levels are relatively good in a majority of participants, while a minority do have clear participation 
restrictions. Measuring participation merits further development to distinguish capacity from performance, and objective indicators from subjective aspects. These issues appear to have different impacts in parent-reports and in children and youth self-reports.

Finally, some behavioral changes after severe TBI, such as apathy (lack of motivation), might have a strong influence on participation and require further investigation. 


\section{References}

[1] Dewan MC, Mummareddy N, Wellons JC, et al. Epidemiology of Global Pediatric Traumatic Brain Injury: Qualitative Review. World Neurosurg. 2016;91:497-509.e1.

[2] Babikian T, Merkley T, Savage RC, et al. Chronic Aspects of Pediatric Traumatic Brain Injury: Review of the Literature. J. Neurotrauma. 2015;32:1849-1860.

[3] Ryan NP, van Bijnen L, Catroppa C, et al. Longitudinal outcome and recovery of social problems after pediatric traumatic brain injury (TBI): Contribution of brain insult and family environment. Int. J. Dev. Neurosci. 2016;49:23-30.

[4] Rosema S, Crowe L, Anderson V. Social Function in Children and Adolescents after Traumatic Brain Injury: A Systematic Review 1989-2011. J. Neurotrauma. 2012;29:1277-1291.

[5] Muscara F, Catroppa C, Eren S, et al. The impact of injury severity on long-term social outcome following paediatric traumatic brain injury. Neuropsychol. Rehabil. 2009;19:541-561.

[6] Chertkoff WN, Owen YK, L WS, et al. Social information processing skills in adolescents with traumatic brain injury: Relationship with social competence and behavior problems. J. Pediatr. Rehabil. Med. Inderdisciplinary Approach. 2009;285295.

[7] Janusz JA, Kirkwood MW, Yeates KO, et al. Social problem-solving skills in children with traumatic brain injury: long-term outcomes and prediction of social competence. Child Neuropsychol. J. Norm. Abnorm. Dev. Child. Adolesc. 2002;8:179-194.

[8] Kapapa T, Pfister U, König K, et al. Head Trauma in Children, Part 3: Clinical and Psychosocial Outcome After Head Trauma in Children. J. Child Neurol. 2010;25:409422.

[9] Ryan NP, Catroppa C, Cooper JM, et al. Relationships between acute imaging biomarkers and theory of mind impairment in post-acute pediatric traumatic brain injury: A prospective analysis using susceptibility weighted imaging (SWI). Neuropsychologia. 2015;66:32-38.

[10] Treble-Barna A, Zang H, Zhang N, et al. Long-Term Neuropsychological Profiles and Their Role as Mediators of Adaptive Functioning after Traumatic Brain Injury in Early Childhood. J. Neurotrauma. 2017;34:353-362.

[11] Levin HS, Hanten G, Li X. The relation of cognitive control to social outcome after paediatric TBI: Implications for intervention. Dev. Neurorehabilitation. 2009;12:320 329.

[12] Hung AH, Cassedy A, Schultz HM, et al. Predictors of Long-Term Victimization After Early Pediatric Traumatic Brain Injury: J. Dev. Behav. Pediatr. 2017;38:49-57.

[13] Katz-Leurer M, Rotem H, Keren O, et al. Recreational physical activities among children with a history of severe traumatic brain injury. Brain Inj. 2010;24:1561-1567. 
[14] Anderson V, Godfrey C, Rosenfeld JV, et al. 10 years outcome from childhood traumatic brain injury. Int. J. Dev. Neurosci. 2012;30:217-224.

[15] Crowe LM, Catroppa C, Babl FE, et al. Intellectual, Behavioral, and Social Outcomes of Accidental Traumatic Brain Injury in Early Childhood. PEDIATRICS. 2012;129:e262e268.

[16] Keenan HT, Clark AE, Holubkov R, et al. Psychosocial and Executive Function Recovery Trajectories One Year after Pediatric Traumatic Brain Injury: The Influence of Age and Injury Severity. J. Neurotrauma. 2018;35:286-296.

[17] Yeates KO, Taylor HG, Walz NC, et al. The family environment as a moderator of psychosocial outcomes following traumatic brain injury in young children. Neuropsychology. 2010;24:345-356.

[18] Lantagne A, Peterson RL, Kirkwood MW, et al. Featured Article: Interpersonal Stressors and Resources as Predictors of Adolescent Adjustment Following Traumatic Brain Injury. J. Pediatr. Psychol. 2018;43:703-712.

[19] Bedell G, Coster W. Measuring Participation of School-Aged Children With Traumatic Brain Injuries: Considerations and Approaches. J. Head Trauma Rehabil. 2008;23:220229.

[20] Morris C. Measuring participation in childhood disability: how does the capability approach improve our understanding? Dev. Med. Child Neurol. 2009;51:92-94.

[21] McCauley SR, Wilde EA, Anderson VA, et al. Recommendations for the Use of Common Outcome Measures in Pediatric Traumatic Brain Injury Research. J. Neurotrauma. 2012;29:678-705.

[22] World Health Organization. International classification of functioning, disability and health. Geneva, Switzerland: WHO; 2001.

[23] Whiteneck G, Dijkers MP. Difficult to Measure Constructs: Conceptual and Methodological Issues Concerning Participation and Environmental Factors. Arch. Phys. Med. Rehabil. 2009;90:S22-S35.

[24] McConachie H, Colver AF, Forsyth RJ, et al. Participation of disabled children: how should it be characterised and measured? Disabil. Rehabil. 2006;28:1157-1164.

[25] Coster W, Khetani MA. Measuring participation of children with disabilities: Issues and challenges. Disabil. Rehabil. 2008;30:639-648.

[26] King G, Law M, King S, et al. A conceptual model of the factors affecting the recreation and leisure participation of children with disabilities. Phys. Occup. Ther. Pediatr. 2003;23:63-90.

[27] Bedell GM. Developing a follow-up survey focused on participation of children and youth with acquired brain injuries after discharge from inpatient rehabilitation. NeuroRehabilitation. 2004;19:191-205. 
[28] Bedell G. Further validation of the Child and Adolescent Scale of Participation (CASP). Dev. Neurorehabilitation. 2009;12:342-351.

[29] Golos A, Bedell G. Psychometric properties of the Child and Adolescent Scale of Participation (CASP) across a 3-year period for children and youth with traumatic brain injury. NeuroRehabilitation. 2016;38:311-319.

[30] Harrison PL, Oakland T. Adaptive Behavior Assessment System. 2nd. Edition. San Antonio: Psychological Corp.; 2003.

[31] Varni JW, Seid M, Rode CA. The PedsQL: measurement model for the pediatric quality of life inventory. Med. Care. 1999;37:126-139.

[32] Golos A, Bedell G. Responsiveness and discriminant validity of the Child and Adolescent Scale of Participation across three years for children and youth with traumatic brain injury. Dev. Neurorehabilitation. 2018;21:431-438.

[33] Chambéron M, Catale C, Kerrouche B, et al. Factors associated with participation in children and adolescents with acquired brain injury: validation of the French version of the Child and Adolescent Scale of Participation (CASP). Disabil. Rehabil. (submitted);

[34] de Kloet AJ, Gijzen R, Braga LW, et al. Determinants of participation of youth with acquired brain injury: A systematic review. Brain Inj. 2015;29:1135-1145.

[35] Anderson V, Beauchamp MH, Yeates KO, et al. Social Competence at Two Years after Childhood Traumatic Brain Injury. J. Neurotrauma. 2017;34:2261-2271.

[36] McDougall J, Bedell G, Wright V. The youth report version of the Child and Adolescent Scale of Participation (CASP): assessment of psychometric properties and comparison with parent report. Child Care Health Dev. 2013;39:512-522.

[37] van Tol E, Gorter JW, DeMatteo C, et al. Participation outcomes for children with acquired brain injury: A narrative review. Brain Inj. 2011;25:1279-1287.

[38] Thompson M, Elliott C, Willis C, et al. Can, Want and Try: Parents' Viewpoints Regarding the Participation of Their Child with an Acquired Brain Injury. Kobeissy FH, editor. PLOS ONE. 2016;11:e0157951.

[39] Bedell GM, Khetani MA, Cousins MA, et al. Parent Perspectives to Inform Development of Measures of Children's Participation and Environment. Arch. Phys. Med. Rehabil. 2011;92:765-773.

[40] Rivara FP, Vavilala MS, Durbin D, et al. Persistence of Disability 24 to 36 Months after Pediatric Traumatic Brain Injury: A Cohort Study. J. Neurotrauma. 2012;29:2499-2504.

[41] Catroppa C, Godfrey C, Rosenfeld JV, et al. Functional Recovery Ten Years after Pediatric Traumatic Brain Injury: Outcomes and Predictors. J. Neurotrauma. 2012;29:2539-2547.

[42] Catroppa C, Hearps S, Crossley L, et al. Social and Behavioral Outcomes following Childhood Traumatic Brain Injury: What Predicts Outcome at 12 Months Post-Insult? J. Neurotrauma. 2017;34:1439-1447. 
[43] Shultz EL, Hoskinson KR, Keim MC, et al. Adaptive functioning following pediatric traumatic brain injury: Relationship to executive function and processing speed. Neuropsychology. 2016;30:830-840.

[44] Anderson V, Beauchamp MH, Yeates KO, et al. Social competence at 6 months following childhood traumatic brain injury. J. Int. Neuropsychol. Soc. JINS. 2013;19:539-550.

[45] Meadows EA, Owen Yeates K, Rubin KH, et al. Rejection Sensitivity as a Moderator of Psychosocial Outcomes Following Pediatric Traumatic Brain Injury. J. Int. Neuropsychol. Soc. 2017;23:451-459.

[46] Teasdale G, Jennett B. Assessment of coma and impaired consciousness. A practical scale. Lancet. 1974;2:81-84.

[47] Baker SP, O’Neill B, Haddon W, et al. The injury severity score: a method for describing patients with multiple injuries and evaluating emergency care. J. Trauma. 1974;14:187-196.

[48] Carney NA, Chesnut R, Kochanek PM. Guidelines for the acute medical management of severe traumatic brain injury in infants, children, and adolescents: Pediatr. Crit. Care Med. 2003;4:1-75.

[49] Chevignard M, Toure H, Brugel DG, et al. A comprehensive model of care for rehabilitation of children with acquired brain injuries. Child Care Health Dev. 2010;36:31-43.

[50] Tepas JJ, Mollitt DL, Talbert JL, et al. The pediatric trauma score as a predictor of injury severity in the injured child. J. Pediatr. Surg. 1987;22:14-18.

[51] Baker SP, O’Neill B. The injury severity score: an update. J. Trauma. 1976;16:882-885.

[52] Copes WS, Champion HR, Sacco WJ, et al. The Injury Severity Score revisited. J. Trauma. 1988;28:69-77.

[53] Beers SR, Wisniewski SR, Garcia-Filion P, et al. Validity of a Pediatric Version of the Glasgow Outcome Scale-Extended. J. Neurotrauma. 2012;29:1126-1139.

[54] Ewing-Cobbs L, Bloom DR, Prasad MR, et al. Assessing Recovery and Disability After Physical Trauma: The Pediatric Injury Functional Outcome Scale. J. Pediatr. Psychol. 2014;39:653-665.

[55] Wilson JTL, Pettigrew LEL, Teasdale GM. Structured Interviews for the Glasgow Outcome Scale and the Extended Glasgow Outcome Scale: Guidelines for Their Use. J. Neurotrauma. 1998;15:573-585.

[56] Wechsler D. Échelle d'intelligence de Wechsler pour enfants: WISC-IV. Paris, France: Les Éditions du Centre de Psychologie Appliquée; 2005.

[57] Wechsler D. Échelle d'intelligence de Wechsler pour adultes: WAIS-IV. Paris, France: Les Éditions du Centre de Psychologie Appliquée; 2011. 
[58] Gioia, GA, Isquith, PK, Guy, SC, et al. Behavior Rating Inventory of Executive Function. Lutz, FL.: Psychological Assessment Ressources, Inc.; 2000.

[59] Roth RM, Gioia GA, Isquith PK. BRIEF-A Inventaire d'évaluation comportementale des fonctions exécutives - version adulte [BRIEF-A Behavior rating inventory of executive function - adult version]. Besnard J, Fournet N, Lancelot C, et al., editors. Adaptation Française. Paris, France: Hogrefe; 2015.

[60] Achenbach TM. Manual for Child Behavior Checklist/ 4-18 and 1991 Profile. Burlington, VT: University of Vermont, Dept. of Psychiatry; 1991.

[61] Varni JW, Limbers CA. The PedsQL 4.0 Generic Core Scales Young Adult Version: feasibility, reliability and validity in a university student population. J. Health Psychol. 2009;14:611-622.

[62] Varni, Burwinkle TM, Katz ER, et al. The PedsQL in pediatric cancer: reliability and validity of the Pediatric Quality of Life Inventory Generic Core Scales, Multidimensional Fatigue Scale, and Cancer Module. Cancer. 2002;94:2090-2106.

[63] Varni JW, Limbers CA. The PedsQL ${ }^{\mathrm{TM}}$ Multidimensional Fatigue Scale in young adults: feasibility, reliability and validity in a University student population. Qual. Life Res. 2008;17:105-114.

[64] Varni JW, Burwinkle TM, Szer IS. The PedsQL Multidimensional Fatigue Scale in pediatric rheumatology: reliability and validity. J. Rheumatol. 2004;31:2494-2500.

[65] Varni JW, Limbers CA, Bryant WP, et al. The PedsQL Multidimensional Fatigue Scale in type 1 diabetes: feasibility, reliability, and validity. Pediatr. Diabetes. 2009;10:321328.

[66] Varni JW, Limbers CA, Bryant WP, et al. The PedsQL multidimensional fatigue scale in pediatric obesity: feasibility, reliability and validity. Int. J. Pediatr. Obes. IJPO Off. J. Int. Assoc. Study Obes. 2010;5:34-42.

[67] Speranza M, Guénolé F, Revah-Levy A, et al. The French version of the Family Assessment Device. Can. J. Psychiatry Rev. Can. Psychiatr. 2012;57:570-577.

[68] Statistical Analysis Software Institute Inc. SAS/STAT ® 9.1 User’s Guide. Cary, NC: Statistical Analysis Software Institute Inc.; 2004.

[69] Marin RS. Differential diagnosis and classification of apathy. Am. J. Psychiatry. 1990;147:22-30.

[70] Marin RS, Fogel BS, Hawkins J, et al. Apathy: a treatable syndrome. J. Neuropsychiatry Clin. Neurosci. 1995;7:23-30.

[71] Arnould A, Rochat L, Azouvi P, et al. A multidimensional approach to apathy after traumatic brain injury. Neuropsychol. Rev. 2013;23:210-233.

[72] Galvin J, Froude EH, McAleer J. Children's participation in home, school and community life after acquired brain injury. Aust. Occup. Ther. J. 2010;57:118-126. 
[73] Aaro Jonsson CC, Emanuelson IM, Charlotte Smedler A. Variability in quality of life 13 years after traumatic brain injury in childhood. Int. J. Rehabil. Res. 2014;37:317-322.

[74] Wells R, Minnes P, Phillips M. Predicting social and functional outcomes for individuals sustaining paediatric traumatic brain injury. Dev. Neurorehabilitation. 2009;12:12-23.

[75] Catroppa C, Crossley L, Hearps SJC, et al. Social and behavioral outcomes: pre-injury to six months following childhood traumatic brain injury. J. Neurotrauma. 2015;32:109115.

[76] Bedell GM, Dumas HM. Social participation of children and youth with acquired brain injuries discharged from inpatient rehabilitation: a follow-up study. Brain Inj. 2004;18:65-82.

[77] Foo WS, Galvin J, Olsen J. Participation of children with ABI and the relationship with discharge functional status. Dev. Neurorehabilitation. 2012;15:1-12. 
Table 1. Pre-injury education, injury severity, 1 year and 7 years post-injury outcomes of the participants in the TBI group

Severe TBI

$(n=37)$

Mean (SD) [Range]

\section{Pre-injury education}

Assisted and/or Delayed, n (\%)

\section{Injury Severity}

Age at injury (years)

Lowest Glasgow Coma Scale Score

Paediatric Trauma Score

Injury Severity Score

Length of coma (days)

1-year post-injury outcome

Overall disability (GOS Peds), $\mathrm{n}(\%)$

Good Recovery

$5(13.51)$

Moderate Disability

$20(54.05)$

Severe Disability

$12(32.43)$

Functional outcome (PIFOS score, $n=31$ )

\section{7-years post-injury outcome}

Overall disability (GOS-E/GOS-E Peds), n (\%)

Good Recovery

Upper level

Lower level

Moderate Disability

Upper level

Lower level

Severe Disability

Upper level

Lower level

\section{Motor deficits}

Presence, n (\%)
$7.62(4.54)[.25-14.67]$

$5.92(1.66)[3-8]$

$3.97(2.34)[-1-+9]$

$27.76(9.72)$ [4 - 50]

6.68 (4.96) [1 - 22]

TBI: Traumatic Brain Injury; SD: Standard Deviation; GOS Peds: Pediatric Glasgow Outcome Scale; GOS-E/GOS-E Peds: Glasgow Outcome Scale-Extended; Glasgow Outcome Scale-Extended, Pediatric version; PIFOS: Pediatric Injury Functional Outcome. 
Table 2. Group comparisons between participants with TBI and controls

\begin{tabular}{|c|c|c|c|c|c|c|}
\hline & \multicolumn{2}{|r|}{$\begin{array}{c}\text { Severe TBI } \\
\quad(n=37)\end{array}$} & \multicolumn{2}{|r|}{$\begin{array}{c}\text { Controls } \\
(n=33)\end{array}$} & \multirow[b]{2}{*}{ Statistic } & \multirow[b]{2}{*}{$p^{\dagger}$} \\
\hline & $n$ & Mean (SD) [Range] & $n$ & Mean (SD) [Range] & & \\
\hline \multicolumn{7}{|l|}{ Sociodemographic characteristics } \\
\hline Age at follow-up & & $15.4(4.4)[7.4-22.7]$ & & $15.3(4.5)[7.2-22.5]$ & $Z=-.2$ & .88 \\
\hline Gender, male, n $(\%)$ & & $23(62.2)$ & & $20(60.6)$ & & \\
\hline \multicolumn{7}{|l|}{ Parental education level, $\mathrm{n}(\%)$} \\
\hline Medium-high & & $19(51.4)$ & & $21(63.6)$ & $X^{2}=1.1$ & .30 \\
\hline \multicolumn{7}{|l|}{ Family situation, n (\%) } \\
\hline Non-marital or single parent & & $12(32.4)$ & & $13(39.4)$ & $X^{2}=.4$ & .55 \\
\hline \multicolumn{7}{|l|}{ 7-years post-injury outcome } \\
\hline On-going education & 37 & & 33 & & & \\
\hline Mainstream, n (\%) & & $29(78.4)$ & & $33(100)$ & $X^{2}-70$ & $005 \%$ \\
\hline Specialized, n (\%) & & $8(21.6)$ & & $0(0)$ & $x=1.9$ & .005 \\
\hline FSIQ & 34 & $86.2(18.5)[40$ - 129] & 33 & $98.9(13.4)[63-124]$ & $Z=3.3$ & .0009 \\
\hline BRIEF (Parent-report) & 31 & & 27 & & & \\
\hline Behavioral Regulation Index & & $60.8(18.1)[35-99]$ & & $48.8(8.5)[37-69]$ & $Z=-2.5$ & .013 \\
\hline Metacognition Index & & $58.1(12.2)[37-81]$ & & $49.8(11.5)[37-72]$ & $Z=-2.5$ & .013 \\
\hline Global Executive score & & $60.3(14.8)[37-89]$ & & $50.2(10.7)[37-73]$ & $Z=-2.7$ & .008 \\
\hline CBCL (Parent-report) & 22 & & 19 & & & \\
\hline Internalizing problems & & $58.7(10.9)[34-71]$ & & $56.5(9.6)$ [33 - 72] & $Z=-.95$ & .34 \\
\hline Externalizing problems & & $55.8(11.9)[33-69]$ & & $48.3(7.3)[34-66]$ & $Z=-2.4$ & .019 \\
\hline Total problems & & $58.9(10.7)[39-76]$ & & $49.3(9.5)$ [25 - 69] & $Z=-2.7$ & .008 \\
\hline \multicolumn{7}{|l|}{ PedsQL } \\
\hline Total score (Parent-report) & 25 & 71.7 (18.9) [34.8 - 96] & 20 & 83 (17) [47.8 - 100] & $Z=2.2$ & .029 \\
\hline Total score (Self-report) & 34 & $70.6(18.2)$ [34.8 - 98.9] & 33 & $83.9(12.7)[52.2-100]$ & $Z=3.3$ & .001 \\
\hline \multicolumn{7}{|l|}{ MFS } \\
\hline Total score (Parent-report) & 25 & $67.8(20.3)[31.9-100]$ & 20 & $87.6(11.4)[58.3-100]$ & $Z=3.4$ & .0006 \\
\hline Total score (Self-report) & 34 & $60.1(18.1)[27.8-90.3]$ & 33 & $77.4(15.3)[41.7$ - 97.2] & $Z=3.7$ & .0002 \\
\hline \multicolumn{7}{|l|}{ FAD } \\
\hline Family functioning & 35 & $1.8(.4)[1-2.7]$ & 33 & $1.6(.6)[0-2.6]$ & $Z=-1.5$ & .13 \\
\hline
\end{tabular}


Table 3. Group comparisons between the TBI and the control groups on the parent and self-report mean scores of the Child and Adolescent Scale of Participation 7 years post-injury

\begin{tabular}{|c|c|c|c|c|c|c|c|c|c|c|}
\hline & \multicolumn{4}{|c|}{ Severe TBI } & \multicolumn{4}{|c|}{ Controls } & \multicolumn{2}{|c|}{ Wilcoxon test } \\
\hline & $\mathbf{N}$ & Mean & SD & Range & $\mathbf{N}$ & Mean & SD & Range & $Z$ & $p^{\dagger}$ \\
\hline \multicolumn{11}{|l|}{ Child and Adolescent Scale of Participation (CASP) } \\
\hline Parent-report & 37 & & & & 31 & & & & & \\
\hline Home participation & & 89.79 & 11.23 & $62.5-100$ & & 95.97 & 6.12 & $79.2-100$ & 2.5 & .014 \\
\hline Neighborhood and community participation & & 85.46 & 17.05 & $31.3-100$ & & 94.97 & 9.18 & $68.8-100$ & 2.7 & .007 \\
\hline School participation & & 93.44 & 10.12 & $65-100$ & & 99.03 & 3.75 & $80-100$ & 3.1 & .002 \\
\hline Home and community living activities & & 81.89 & 18.48 & $30-100$ & & 94.15 & 7.41 & $75-100$ & 2.9 & .004 \\
\hline Total Score & & 86.39 & 14.81 & $31.7-100$ & & 96.35 & 4.76 & $82.5-100$ & 3.6 & .0004 \\
\hline Self-report & 27 & & & & 26 & & & & & \\
\hline Home participation & & 90.62 & 9.16 & $66.7-100$ & & 91.03 & 9.62 & $66.7-100$ & .2 & .87 \\
\hline Neighborhood and community participation & & 87.78 & 14.34 & $50-100$ & & 87.93 & 15.68 & $43.3-100$ & .3 & .78 \\
\hline School participation & & 93.18 & 11.08 & $60-100$ & & 94.42 & 10.89 & $60-100$ & -.9 & .34 \\
\hline Home and community living activities & & 85.19 & 16.90 & $40-100$ & & 91.78 & 14.17 & $35-100$ & 1.8 & .08 \\
\hline Total Score & & 89.12 & 10.56 & $62.5-100$ & & 91.24 & 9.61 & $66.3-100$ & .8 & .44 \\
\hline
\end{tabular}

Two-tailed Wilcoxon signed-rank test (Z); TBI: Traumatic Brain Injury; SD: Standard deviation. 
90

80

70

60

50

40

30

20

10

0

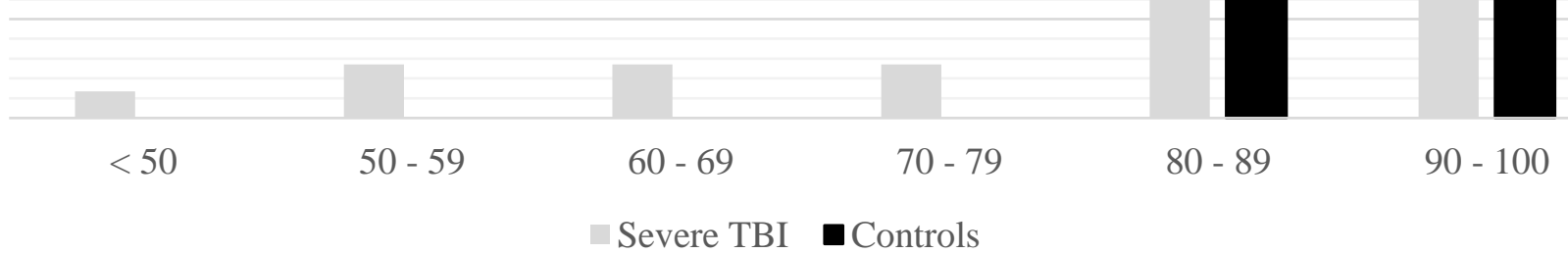

Figure 1. Distribution of CASP Parent Total scores in controls and participants with severe TBI 7-years postinjury. Lowest score observed in the control group $=82.5$. Eight patients $(22 \%)$ presented scores lower than 82.5 . 